\title{
On the Performance of the Intel SR30 Depth Camera: Metrological and Critical Characterization
}

\author{
Monica Carfagni, Rocco Furferi, Lapo Governi, Michaela Servi, Francesca Uccheddu, and Yary Volpe
}

\begin{abstract}
Specifically conceived for applications related to face analytics and tracking, scene segmentation, hand/finger tracking, gaming, augmented reality, and RGB-D cameras are nowadays used even as 3-D scanners. Despite depth cameras' accuracy and precision are not comparable with professional 3-D scanners, they still constitute a promising device for reverse engineering (RE) applications in the close range, due to their low cost. This is particularly true for more recent devices, such as, for instance, the RealSense SR300, which promises to be among the best performing close range depth cameras in the market. Given the potentiality of this new device, and since to date a deep investigation on its performances has not been assessed in scientific literature, the main aim of this paper is to characterize and to provide metrological considerations on the Intel RealSense SR300 depth sensor when this is used as a 3-D scanner. To this end, the device sensor performances are first assessed by applying the existing normative guidelines (i.e. the one published by the Association of German Engineers - Verein Deutscher Ingenieure VDI/VDE 2634) both to a set of raw captured depth data and to a set acquired with optimized setting of the camera. Then, further assessment of the device performances is carried out by applying some strategies proposed in the literature using optimized sensor setting, to reproduce "real life" conditions for the use as a 3D scanner. Finally, the performance of the device is critically compared against the performance of latest short-range sensors, thus providing a useful guide, for researchers and practitioners, in an informed choice of the optimal device for their own RE application.
\end{abstract}

Index Terms-SR300, depth camera, device characterization, VDI/VDE normative, structured light, temporal multiplexing.

\section{INTRODUCTION}

$\mathbf{P}$ ORTABLE 3D optical measurement is a growing segment in the metrology landscape, with new technologies and approaches being introduced continuously. 3D data acquisition and processing have been restricted for many years to the academic sector and industrial applications, but the recent introduction of consumer-accessible depth cameras (named also range cameras and RGB-D cameras) has opened wide new interesting possibilities of 3D computer vision applications.

Due to the high framerates, depth cameras represent an attractive option for a number of industrial applica-

Manuscript received March 22, 2017; revised May 8, 2017; accepted May 8, 2017. Date of publication May 16, 2017; date of current version June 23, 2017. The associate editor coordinating the review of this paper and approving it for publication was Prof. Danilo Demarchi. (Corresponding author: Francesca Uccheddu.)

The authors are with the Department of Industrial Engineering, University of Florence, 50100 Firenze, Italy (e-mail: monica.carfagni@unifi.it; rocco.furferi@unifi.it; lapo.governi@unifi.it@unifi.it; michaela.servi@unifi.it; francesca.uccheddu@unifi.it; yary.volpe@unifi.it).

Digital Object Identifier 10.1109/JSEN.2017.2703829 tions. Simultaneous localization and mapping (SLAM) [1], dense 3D mapping [2], hand gesture, pose recognition and Reverse Engineering (RE) are only few examples of possible applications. Even if the performance of RGB-D cameras is known to be lower than the one of professional 3D scanners, the low cost of these devices makes them still attractive for RE field, as demonstrated by a number of works proposed in literature [3], [4]. Consequently, the performance assessment of RGB-D cameras when used as a 3D scanner is nowadays a hot topic in the sensors field.

It is worth noting that the main available consumer depth camera models, occupy different scan ranges with some overlap also in terms of possible applications. A possible classification of depth cameras on the basis of their working range divides such devices into three categories: mid-to-long range $(>1.5 \mathrm{~m})$, close range $(0.1 \mathrm{~m}$ to $1.5 \mathrm{~m})$ and nano/micro range $(<10 \mathrm{~mm})$.

The Microsoft Kinect ${ }^{\mathrm{TM}}$ [5] is the first consumer depth camera product, based on the Primesensor ${ }^{\mathrm{TM}}$ design by Primesense (Israel) [6]. The same design also appeared in other consumer products, such as the Asus Xtion [7] (ASUSTeK Computer Inc., Taipei, Taiwan) and the Occipital Structure Sensor [8]. The same technology has been then embedded in the Astra Pro 3D (by ORBBECTM [9]). Intel (Intel Corporation, Santa Clara, California) has instead released the "Realsense" family, a competitive triangulation-based range sensing technology [10], implemented in standalone units by Creative (Creative Technology, Singapore), and in some Lenovo (Lenovo Group Limited, China) laptops.

Among the new Intel® RealSense ${ }^{\mathrm{TM}}$ sensors family, the SR300 depth sensor can be considered one of the most promising devices. This sensor has the potential to partially overcomes the limitation of other commercially available temporal based depth camera systems, e.g. by leveraging the compactness and efficiency offered by micro-electro-mechanical mirror based laser projector and by faster sensors.

Given the potentiality of this new device, and since to date a deep investigation on its performances has not been assessed in scientific literature, the main aim of the present work is to characterize and to provide metrological considerations on the Intel ${ }^{\circledR}$ RealSense ${ }^{\mathrm{TM}}$ SR300 depth sensor when this is used as a 3D scanner.

To this end, the device sensor performances in terms of accuracy of indication of size and form, are firstly assessed by applying the VDI/VDE normative (i.e. the international standard for characterization of measuring devices) to a 
set of raw captured depth data. Limiting the analysis to the raw data extracted only by the depth sensor allows to discard possible signal processing of the many filters available on the released cross-platform camera capture for Intel ${ }^{\circledR}$ RealSense ${ }^{\mathrm{TM}}$. In other words, the analyzed sensor data are processed using the normative without optimizing it so as to detect the worst possible conditions a user might incur in using the SR300 device.

Further assessment of the device performances is carried out by using "real life" setting (i.e. selecting an optimized profile for the use as a 3D scanner) and by applying best practices proposed in literature. Even if in this case it is practically impossible to decouple sensor performance from signal processing algorithm performance, we think this analysis is extremely useful for users willing to adopt this camera as a low-cost 3D scanner.

Additionally, the performance of the device is critically compared against the performance of latest short-range devices, thus providing a useful guide, for researchers and practitioners, in an informed choice of the optimal device for their own RE application.

The main issue related to the performance assessment of the above-mentioned devices, is related to the lack of a comprehensive internationally-recognized standard (or a guideline) helpful in defining a common language between users and manufacturers.

As such, the inadequacy of standard characterization methods from trusted sources make it difficult for a user to select a device among others, based on the specification sheet provided by the manufacturer. This is particularly true for close range systems where the gap in the existing documentary and physical standards is more evident. Examples of this gap are related to the lack of methods for performance assessment on freeform surfaces, for accuracy of 3D moving cameras acquiring static objects, or for the accuracy of 3D static cameras acquiring moving reference [11]. According to the considerations made above, international standards can be only partially applied to assess the performance of the Intel® SR300 depth camera, resulting inadequate to discover all its potential. Therefore, in the present work standard procedures commonly used for measuring devices are complemented with practices proposed in scientific literature.

The paper is organized has follows. Section 2 presents a review of both standardized and non, close-range camera characterization parameters, together with the Intel SR300 depth camera specifications and working principle. Section 3 will report the results of SR300 performance test with raw data, the systematic error evaluation using the optimized options for the camera when the intended use is as a 3D scanner and multiple views 3D scanning evaluation compared with similar close range depth camera systems. Results are finally discussed and conclusions drafted in Section 4.

\section{MAterials AND Methods}

The measurement range in close range applications may vary from few centimeters to one meter. The trustworthiness of depth camera systems is crucial to determine whether the acquired data fits the specific application requirements.
The purpose specifications are also important in defining test methods that highlights impartial weaknesses and strengths of the systems. To fairly quantify the overall accuracy of the 3D optical imaging system, appropriate physical standards, together with related tests procedures, are needed. The goal of a standard is to provide a way to evaluate product performance for a specific need. Close range, structured light based scanners, need to be verified over their close-range volume and often, over multiple registered 3D images representing a 360 degrees' object surface.

In this section, test objects and procedures recommended by the existing guidelines to metrologically characterize 3D imaging system are discussed. It is worth noting again that since not all the desirable performance assessments are addressable by following the guidelines, we discuss also the alternative/ integrating approaches devised in the metrologic community. In the last subsection are reported the description and the main characteristics of the SR300 depth sensor.

\section{A. VDI/VDE Normative}

The only available short-range 3D imaging standard today is the German standard VDI/VDE 2634 [12]. The specific guidelines for the acceptance and reverification of optical based 3D scanning systems cover the following issues:

Part 1: "Imaging systems with point-by-point probing";

Part 2: "Optical systems based on area scanning";

Part 3: "Multiple view systems based on area scanning";

Since the VDI/VDE 2634 is derived from coordinate measuring machines $(\mathrm{CMM})$, it naturally considers measurements at close range.

A comprehensive report on standards developments of 3D imaging systems is reported in [11]. VDI/VDE guideline defines a method of measuring a reference object (sphere, plane), which is typically used to define some basic feature of the analyzed optical system. To assure traceability of 3D measurements of a specific device, acceptance test and re-verification are univocally defined.

Acceptance test involves the measurement of a calibrated artefact; the test is accepted if the error lies inside the limits specified by the manufacturer, while the re-verification test is a repetition of the acceptance test over time.

The VDI/VDE 2634 also recommends the artefacts, the procedure and the method to calculate the results, and the interpretation of the results. VDI/VDE defines three types of standard object: a sphere, a ball bar and a plane; some examples of calibration standards are shown in Fig. 1 and the recommended arbitrary positions are illustrated in Fig. 2.

The guidelines propose to use diffuse reflecting test objects materials, certified with a known level of accuracy and uncertainty (4-5 times better than the device under test).

The considered operations are performed on a single point cloud (as described in Part 2) or on a multi-view registered point cloud (as described in Part 3); the normative also allows the measured values be filtered and pre-processed only if they are part of the boundary conditions for the characteristic test, or it is a routine event in the operation of the system.

Being $\mathrm{L}_{0}$, the main diagonal length of the scanning volume, the sphere diameter must be between 0.1 and 0.2 times $L_{0}$. 


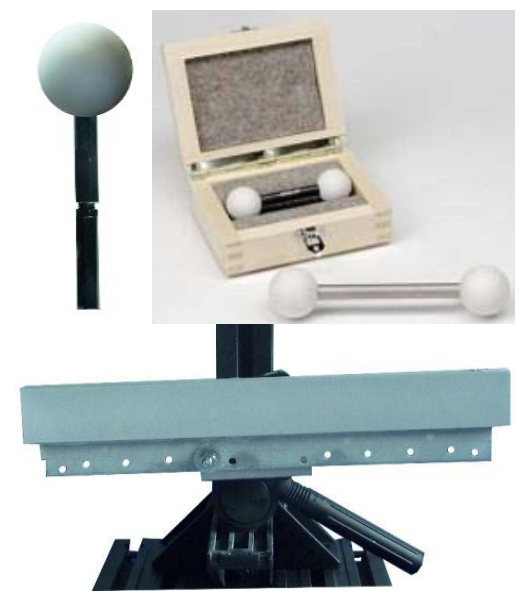

Fig. 1. Examples of 3D calibration standards for probing error and flatness error calculation according to VDI/VDE 2634.
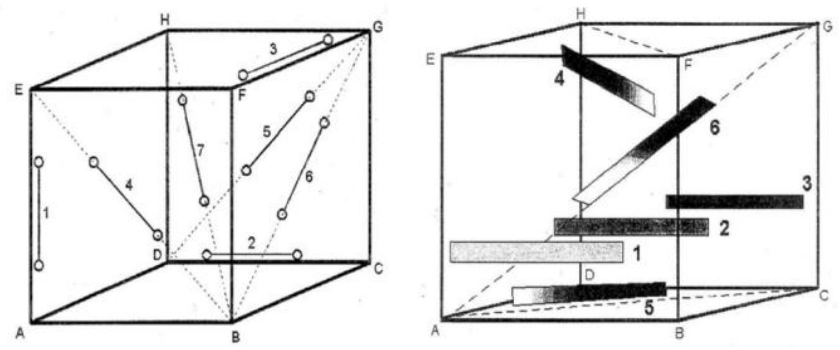

Fig. 2. Recommended arbitrary positions within the systems field of view covering the working volume.

The characteristic estimated with such a sphere is the Probing Error $\mathrm{P}$ that describes the characteristic error of the system within a small portion of the measuring volume attempting to evaluate the intrinsic properties of the $3 \mathrm{D}$ imaging sensor. The form probing error is the range of radial distance between the real measured points and a best-fit sphere evaluated according to the least-squares method. The size probing error is the difference between the estimated (as the best fit sphere) and the "true" diameter of the sphere. The guidelines recommend to survey at least ten uniformly-distributed positions of the artefact within the operative volume.

The ball-bar is a bar connecting two spheres. The distance among the ball centres is $\mathrm{L}_{\mathrm{p}}$ and must be larger or equal to $0.3 \cdot \mathrm{L}_{0}$. The characteristic estimated with this artefact is the Sphere Spacing Error SD that proves the capability of the system in measuring lengths. SD is the difference between the acquired distance and the "true" distance between the centres of the two spheres (estimated from the point cloud of data using a best-fit sphere-fitting); the guidelines recommend to survey at least seven positions within the operative volume.

The plane is a rectangular parallelepiped wide at least $50 \mathrm{~mm}$ and long not less than $0.5 \cdot \mathrm{L}_{0}$. The characteristic estimated with this artefact is the Flatness Measurement Error $F$ that is the range of the signed distances of the measured points from the best-fit plane; the guidelines recommend to measure at least six different orientations of the artefact.

The normative indicates that only $0.3 \%$ of the worst data can be rejected for such test. However, no indication about the number of points needed for the test is reported. Therefore, the results cannot be compared fairly between different types of scanners or other types of measurement instruments.

The more recent part of the VDI/VDE 2634 (Part 3) is dedicated to the most common scenario found in many applications: the multiple $3 \mathrm{D}$ views. The $3 \mathrm{D}$ views are commonly aligned each other in the same coordinate system through an ICP algorithm [13]. The probing error is affected by two influence factors: the errors of a single 3D image (as found per Part 2 tests) and the errors due to the transformation of the different 3D images in a unique coordinate system. As for the Part 2, both form and size describe the quality parameter. The sphere artefact is suggested to be surveyed in at least three arbitrary positions within the operative volume, and for each of them, the artefact must be measured from at least five sensor positions to fully capture the surface.

The sphere-spacing error is used to test the capability of the complex system of performing length measurements. The error is computed using ball bars, ball beams, gauge blocks, step gauges or ball plates. The actual procedure requires a reference artefact that is fully characterised, i.e., the calibration certificate must contain both the spacing and the form of all probing elements and the roughness of the surfaces should be negligibly small. The reference artefact is measured in seven different positions.

\section{B. Other Approaches Provided in Literature}

The scientific community has been working worldwide on possible characterization protocols to assess the devices performances and comparing each other in many operating fields: new parameters has been investigated, new standard artefacts proposed as well as new procedures devised. Gonzalez-Jorge et al. [14] proposed a metrological comparison between Kinect v1 and Kinect v2 by using a certified standard artefact developed at University of Vigo based on 5 spheres and 7 cubes. The accuracy and precision tests are done for different ranges and changing the inclination angle between each sensor and the artefact. Wasenmüller et al. [15] investigated the accuracy and precision of the Kinect v1 and v2 for their usage in the context of 3D reconstruction, SLAM or visual odometry. They based the accuracy and precision of the devices on a ground truth (a planar wall) detected through the pose estimation of a checkerboard on the wall. They tested the devices performances in terms of varying temperature, camera distance and object color.

Zennaro et al. [16], compared the depth data that can be obtained using two depth camera sensors, pointing out the devices performance in terms of accuracy and robustness to artificial illumination and sunlight. The same result of range depending accuracy is found by Khoshelham and Elberink [17] where, to investigate the systematic errors in Kinect data, a comparison is accomplished with a point cloud (the ground truth) obtained by a high-end laser scanner. The first generation of Microsoft device is also investigated by Dutta [18] to determine whether the hardware sensing components were sensitive enough to be used as a portable 3D motion capture system for workplace ergonomic assessments. Dutta compares the acquisition of four cubes obtained with both Kinect v1 and 


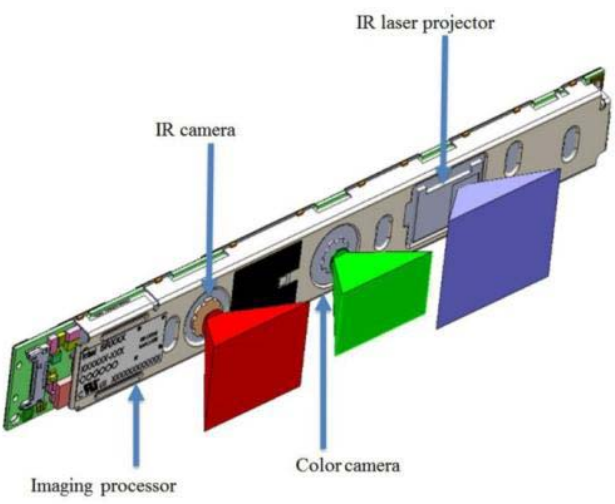

Fig. 3. Components location inside the camera.

a Vicon motion capture system (considered as the ground truth). Menna et al. in [24] evaluate the Kinect accuracy in modelling indoor environments by measuring a special rigid test-field with a calibrated reference scale bar.

The suitability and accuracy of RGB-D cameras for close range 3D modelling was investigated by Lachat et al. [19]; their study highlights errors arising from the environment and the properties of the captured scene and reflectivity of the items. A model automatically obtained using an appositely devised tool is compared to a ground truth model. Breuer et al. [20] also provided an analysis of measurement noise, accuracy and other error sources with the Kinect v2, pointing out a significant distance dependent error and that the device is capable of better resolving fine structures in certain scenarios although the image resolution of the depth camera has been decreased. Guidi et al. [21] proposed a simple yet effective method for estimate the systematic error that once modelled can be eliminated through a proper calibration. The 3D digitizer is considered as a "black box" system whose behavior influence the 3D cloud differently at different spatial frequencies. Given that the system uncertainty is given by random and systematic errors, they model the global random contribution of each range image of a reference plane as Additive White Gaussian Noise (AWGN) that is associated to high spatial frequency. By means of a smoothing filter they filter out the random errors thus estimating the systematic error.

\section{Intel@ SR300 Depth Camera}

The SR300 is the second generation of front-facing Intel ${ }^{\circledR}$ RealSense ${ }^{\mathrm{TM}}$ cameras. It is a subassembly camera product that implements a short range (SR), coded light, 3D imaging system (Fig. 3).

Along with an infrared laser projector, the subassembly includes a Fast VGA infrared camera and a 2-M pixel RGB color camera with an integrated image signal processor. Fast VGA depth mode reduces exposure time and allows dynamic motion up to $2 \mathrm{~m} / \mathrm{s}$.

This new feature leads to a less-noisy result when the scene is not static, as can be qualitatively seen in Figure 4 where a moving hand, captured respectively with a F200 [22] and SR300 camera, is shown.

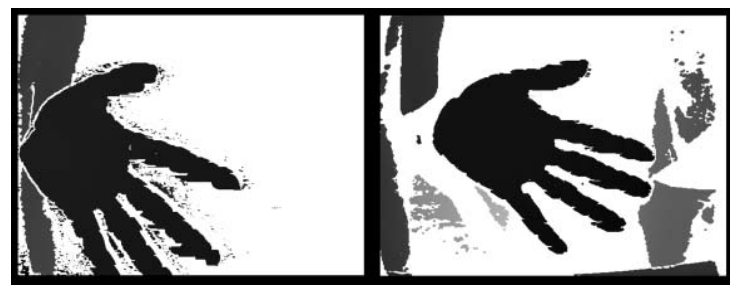

Fig. 4. A single depth frame of a moving hand captures with F200 depth camera (left) and SR300 depth camera (right).

TABLE I

TEChNicAl SpecificAtions OF InTEL REALSENSE SR300

\begin{tabular}{cc}
\hline \hline Technology & CODED LIGHT, FAST VGA 60 FPS \\
\hline Color Camera & UP TO 1080P 30 FPS, 720P 60 FPS \\
Indoor Range & $20-150$ CM \\
Depth FOV (D x W x H) & $88+/-3^{\circ} \times 71.5+/-2^{\circ} \times 55+/-2^{\circ}$ \\
Depth fps & $30 / 60$ \\
IR fps & $30-300$ \\
\hline \hline
\end{tabular}

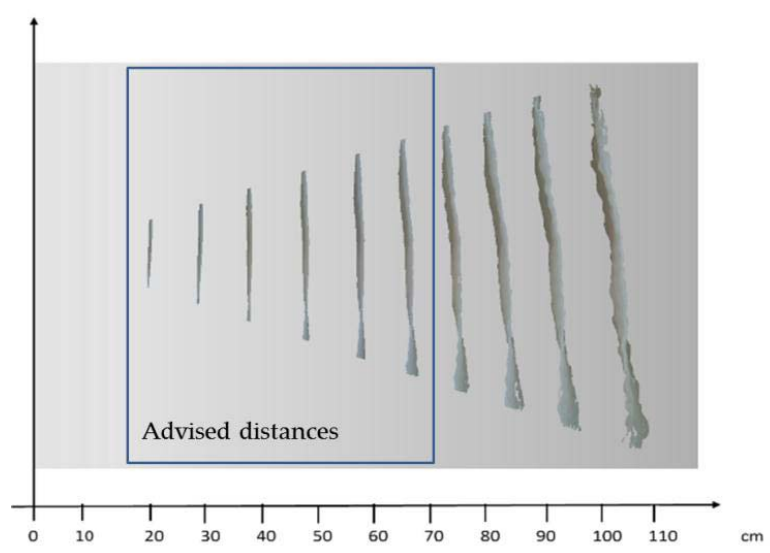

Fig. 5. Qualitative evaluation of the error introduced in measuring a flat surface. Over $70 \mathrm{~cm}$ the acquisition degrades considerably, thus restricting the use of the sensor as a 3D scanner to such distance

The SR300 also provides synchronized color, depth, and IR video streaming to the client system.

The effective range of depth is optimized from $0.2 \mathrm{~m}$ to $1.5 \mathrm{~m}$ for use indoors. The dimensions of the device are approximately $110 \mathrm{~mm} \times 12.6 \mathrm{~mm} \times 3.8-4.1 \mathrm{~mm}$; its weight is $9.4 \mathrm{~g}$, which makes it very compact and well-suited for a wide range of 3D imaging applications.

The manufacturer overall technical specifications of Intel ${ }^{\circledR}$ RealSense SR300 are summarized in Table I. Although the overall camera range is $20-150 \mathrm{~cm}$, the range for using the sensor as a pure 3D scan is suggested to stay within $70 \mathrm{~cm}$.

Beyond such a distance, the accuracy of the camera drastically decreases, as shown in Fig. 5 in which a plane wall portion is captured at incremental distance from the camera, starting from $20 \mathrm{~cm}$ up to $150 \mathrm{~cm}$ with a step of $10 \mathrm{~cm}$ approximately.

The horizontal resolution (usually named "point density") of a 3D optical scanner is defined as the spatial sampling frequency of the area framed in the orthogonal position with respect to the sensor. 
TABLE II

TECHNICAL SPECIFICATIONS OF INTEL $®$ REALSENSE SR300

\begin{tabular}{cccccc}
\hline \hline Distance & $\begin{array}{c}\text { Scale } \\
\text { factor }\end{array}$ & $\mathbf{X}$ & $\mathbf{Y}$ & $\begin{array}{c}\text { Framed } \\
\text { area }\end{array}$ & $\begin{array}{c}\text { Point } \\
\text { density }\end{array}$ \\
\hline$[M M]$ & $\%$ & {$[M M]$} & {$[M M]$} & {$\left[M M^{2}\right]$} & $\begin{array}{c}{[\text { PoINTS/ }} \\
\left.M M^{2}\right]\end{array}$ \\
200 & 0.4 & 256 & 192 & 49152 & 6.25 \\
450 & 1 & 640 & 480 & 307200 & 1.00 \\
600 & 1.3 & 832 & 624 & 519168 & 0.59 \\
800 & 1.8 & 1152 & 864 & 995328 & 0.31 \\
1000 & 2.3 & 1472 & 1104 & 1625088 & 0.19 \\
\hline \hline
\end{tabular}

This area will increase with increasing distance of the sensor from the surface, producing a decrement of the point density. Intel ${ }^{\circ}$ SR300, having a depth camera resolution of $640 \times 480$ pixel and a depth field of view of diagonal $88^{\circ} \times$ width $71.5^{\circ} \times$ height $55^{\circ}$, makes no exception as demonstrated in Table II where point density measured at increasing distances of the sensor from the scene is listed.

The table has been populated computing the scale factor as follows in which the scale factor of the depth image at different distance is given by the following relation:

$$
\text { scalefactor }=\frac{\operatorname{sen}(\beta) *(d)}{\operatorname{sen}(\delta) * \# \text { pixels }}
$$

where $\mathrm{d}$ is the camera-subject distance, $\beta$ is half of the opening angle and $\delta$ the angle of incidence of the camera.

The overall performance of a depth camera system is highly influenced by the used pattern projector illuminator. The Intel ${ }^{\circledR}$ RealSense cameras partially overcomes the limitation of temporal multiplexing [23], [25] illuminators (i.e. unreliable in case of fast moving scenes), by leveraging the innovative MEMS mirror technology [25] to speed up the pattern frames projection time. This is possible thanks to the use of a miniaturized projector made of three tiny components: an IR laser, a line lens and a resonant micro mirror. The line lens takes the point of light from the IR laser and turns it into a flat horizontal line. This is subsequently bounced off the resonant micro mirror, which is twisted by an electrical signal. This micro mirror is moved by a torsional drive system, where an electrostatic signal twists the mirror, which is manufactured in a single piece. The combination of lens and rapidly moving mirrors creates a pattern of light that is projected, and the reflection is detected by the IR camera on the other side of the module, which is used to acquire the scene.

The new RealSense camera provide a rich SDK library that allows to configure the camera with a number of internal settings, such as Accuracy, Filter Option, Laser Power and so on: some of them are post-processing settings, others are acquisition settings; furthermore, a set of default configurations are possible. Such versatility allows users to arrange the best possible setup for the task under study.

\section{EXPERIMENTAL RESULTS}

Data acquisition for the sensor characterization was carried out using a dedicated software, developed by means of the open source library "Librealsense" [26], which is a crossplatform library that allow access to the Intel® Realsense device. We acquired the point cloud after the first 30 frames to allow an initial camera stabilization.

In the following subsections, a description on how the Intel ${ }^{\circledR}$ SR300 overall accuracy has been assessed by following both applicable rules stated in the VDI/VDE standard and literature methods to assess other camera features relevant for close range applications.

As mentioned in the introductory section, the experimental results obtained by applying the VDI/VDE normative are firstly performed by decoupling the contribution coming from the sensor raw data from the contribution of the processing of the many filters available on the released cross-platform camera capture for Intel ${ }^{\circledR}$ RealSense ${ }^{\mathrm{TM}}$. Consequently, the analysed sensor data are processed without optimizing it thus allowing to understand which are the worst performances of the device when used as a 3D scanner. After this preliminary analysis, the VDI/VDE standard has been applied with reference to optimized camera setting, but still taking into account only the data coming from depth sensor (information from RGB sensor are not considered in this work). Then, systematic errors affecting the device (with optimized setting) during 3D acquisition are analysed with the help of a Coordinate Measuring Machine. Finally, a multipleview acquisition of two objects is carried out to qualitatively assess the overall performance of (optimized) device.

\section{A. VDI/VDE-Based Performance Test Using Raw Data}

Inspired by the VDI/VDE 2634 Part 2 recommendations, the device performance test has been carried out first by identifying the auxiliary parameter $\mathrm{L}_{0}$ defined as the body diagonal of the measuring volume. As mentioned above, this parameter is needed for sizing the spheres, the bar and the plane for the performance evaluation test.

The normative suggests a sphere diameter between 0.1 and 0.2 times $\mathrm{L}_{0}$; this translates in the present case, in a diameter between 9.5 and $19 \mathrm{~cm}$. The distance between the spheres in the ball-bar should be larger than $0.3 * \mathrm{~L}_{0}$, i.e. in the present case larger than $28.6 \mathrm{~cm}$. The plane should wide at least $0.5 \mathrm{~cm}$ and long at least $0.5 * \mathrm{~L}_{0}$ thus resulting ranging from $0.5 \mathrm{~cm}$ and $47.6 \mathrm{~cm}$. Accordingly, selected target to be acquired are: 1) a single sphere with diameter of $14 \mathrm{~cm}$; 2) a ball-bar composed of two spheres with diameter equal to $14 \mathrm{~cm}$ fixed at $39.2 \mathrm{~cm}$ from one another and 3) a plane iron bar of dimension $5 \mathrm{~cm} \times 50 \mathrm{~cm}$ (see Fig.6).

The balls material is polystyrene which proves to have optimal diffusive infrared reflection proprieties, even though not certified as suggested by the normative.

To deal with the lack of a certification for the target artefacts, these have been acquired by using a high precision laser scanner, the Romer Absolute Arm 7520 SI/SE (Hexagon Metrology S.p.A., Turin, Italy) that has an overall accuracy of $\pm 0.063 \mathrm{~mm}$, therefore allowing to obtain a sufficiently reliable "reference measure".

In other words, the 3D scanned data obtained with the Romer scanner are considered the ground truth for testing the SR300 device. 

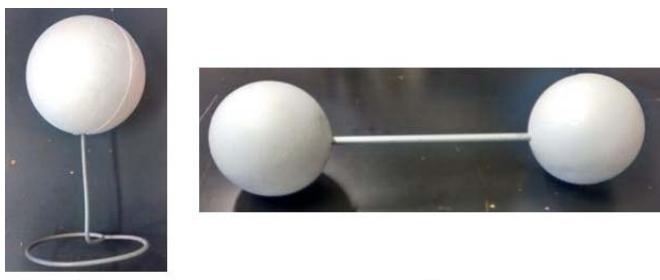

(a)

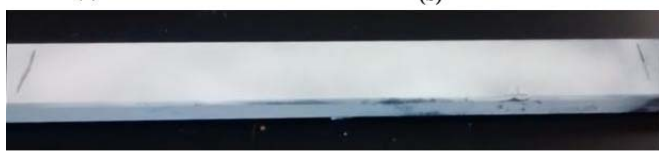

(c)

Fig. 6. Artefacts proposed to measure the quality parameters inspired by the VDI/VDE normative: (a) single sphere having diameter of $14 \mathrm{~cm}$; (b) ball-bar composed of two spheres with radius of $7 \mathrm{~cm}$ fixed at $39.2 \mathrm{~cm}$ from one another; and (c) iron bar of dimensions $5 \times 50 \mathrm{~cm}$.

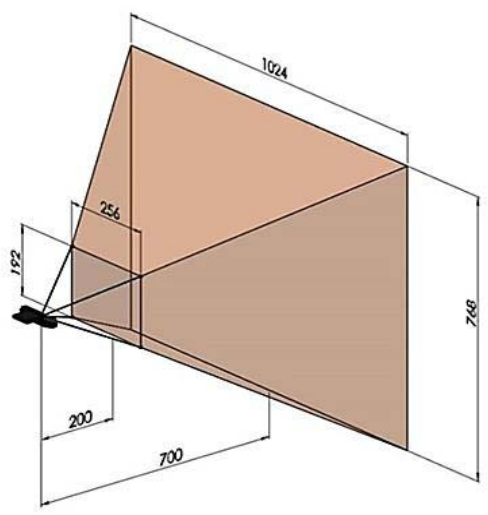

Fig. 7. Truncated pyramid delimitating the working volume boundaries.

The camera is mounted on a stable tripod in front at the cuboid at $20 \mathrm{~cm}$ far from its smallest face. Not to leave any blind area when choosing the reference working volume, for close range 3D acquisitions evaluation, a truncated pyramid having diagonal equal to $953 \mathrm{~mm}$ (see Fig. 7) has been considered as the device actual working volume.

VDI/VDE-based tests have been repeated 4 times using five different units of the SR300 devices to avoid the possibility of testing a defective device. Since the maximum percentage difference, in absolute terms, between two couples of parameters acquired using the devices is within $4 \%$, tests can be considered reliable. Moreover, to correctly measure the normative-suggested parameters we discard points for which the angle between acquisition direction and face normal direction is larger than $75^{\circ}$.

1) Probing Error Evaluation: The first test consists of evaluating the probing error both in terms of form and size. Accordingly, the target sphere has been positioned, in 10 sequential arbitrary locations (as indicated in the normative) within the working volume (see Figure 8a). The form probing error $\left(P_{F i}\right)$ is defined for each acquisition, as the range of radial deviations of the measured spheres points and the best-fit sphere:

$$
P_{F i}=R_{\text {imax }}-R_{\text {imin }}
$$
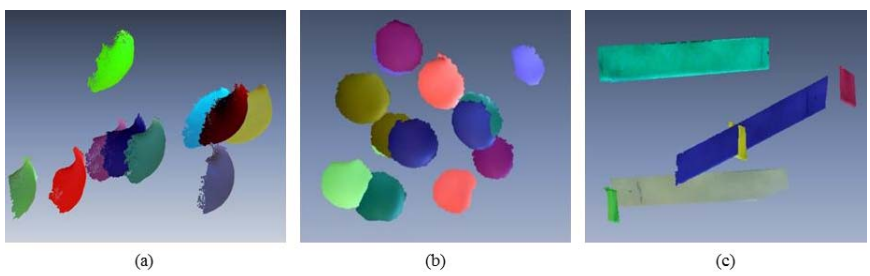

Fig. 8. (a) 3D recording of the single sphere captured in 10 arbitrary positions homogeneously distributed within the working volume. (b) 3D points of the ball-bar captured in 7 positions within the working volume (the same sphere color corresponds to the same bar). (c) 3D points of the plane captured in 6 positions within the working volume.

TABLE III

Results of the Probing ERror Test (Size AND Form), SPHERE-SPACING AND FlatNeSS OBTAINED USING RAW DATA AND DATA ACQUIRED WITH OPTIMIZED PARAMETERS

\begin{tabular}{crccc}
\hline \hline Device & $\boldsymbol{P}_{\boldsymbol{F}}[\mathbf{m m}]$ & $\boldsymbol{P}_{\boldsymbol{S}}[\mathbf{m m}]$ & $\boldsymbol{S S}[\mathbf{m m}]$ & $\mathbf{F}[\mathbf{m m}]$ \\
$\begin{array}{c}\text { SR300 } \\
\text { raw data }\end{array}$ & 15.43 & 4.57 & 5.11 & 19.33 \\
$\begin{array}{c}\text { SR 300 } \\
\text { filtered data }\end{array}$ & 8.30 & 1.91 & 6.05 & 6.88 \\
\hline \hline
\end{tabular}

Where $R_{\text {imax }}$ and $R_{\text {imin }}$ are, respectively, the maximal and minimal distance of the measured surface points of the $i^{t h}$ sphere from the centre of the compensating element (i.e. the best-fit sphere).

The size probing error $\left(P_{S i}\right)$ describes for each acquisition, the difference between the estimated diameter $\left(D_{m i}\right)$ and the "true" diameter $\left(D_{c}\right)$ of the sphere:

$$
P_{S i}=D_{m i}-D_{c}
$$

The estimated diameters are assessed through a best sphere fit process, implemented by the least square method; the "true" diameter, is defined as the one measured on the target spheres using the Romer Absolute Arm 7520 SI/SE, i.e. is the diameter of the ground truth.

From the above defined probing errors, testing form and size of the 10 spheres (repeated 4 times as previously mentioned), it is possible to evaluate the average value of both kind of errors, namely: $P_{F}$ (average form error) and $P_{S}$ (average size error). The absolute values of these errors are listed in Table III (see second row).

2) Sphere-Spacing Error Evaluation: The sphere-spacing error $\Delta l_{i}$ is measured by using the balls-bar target. Such an error is defined as the difference between the acquired distance $l_{m_{i}}$ and the "true" distance $l_{k}$ (i.e. the one measured with the Romer Absolute Arm 7520 SI/SE) between the centres of the two spheres.

$$
\Delta l_{i}=l_{m_{i}}-l_{k}
$$

The target sphere has been positioned, in 7 sequential arbitrary locations (as indicated in the normative) within the working volume (see Figure 8b).

In Table III the averaged value of sphere-spacing and flatness errors are reported. 
3) Flatness Error Evaluation: The flatness (or planarity) error $\left(F_{i}\right)$, is computed as the minimum distance between two parallel planes comprising the $i^{t h}$ scanned point cloud.

To measure this parameter, the plane has been sequentially uniformly-placed, in arbitrary positions inspired by the VDI/VDE normative, within the working volume as shown in Figure 8c.

\section{B. VDI/VDE-Based Performance Test Using Optimized Data}

As mentioned in the introductory section, the main aim of the present work is to assess the performance of the SR300 device when this is used as a 3D scanner. Accordingly, it is worth to repeat the VDI/VDE tests using optimized parameters (as officially distributed in the Intel library [26] for 3D scanning). The chosen parameters are the one provided by the default filter option value that provides moderate smoothing effect to balance between good sharpness level, high accuracy and moderate noise artifacts. Overall results of the repeated test are in Table III.

Results show that the available signal processing algorithms, can greatly improve the overall accuracy of the system except the sphere-spacing error, representing the traceability of the experiment [11].

\section{Systematic Errors}

One of the most common systematic errors for the kind of devices studied in this paper is the so called "distance inhomogeneity" i.e. the set of possible errors coming out from acquisitions at different distances from the sensor.

To study such systematic errors, the camera, set with optimized parameters, is positioned parallel to a white planar wall at different well-known distances to 1) acquire such plane with the 3D device and 2) compare the acquired data with the planar wall. The systematic distance measurement error evaluation has been approached in literature [19], [21], [27] through custom made set up by manually positioning the camera on tripods "parallel" to a wall then moving the tripod.

In this study the perpendicular alignment with the optical axis direction and the acquisition of the planar target (with certified flatness of $3 \mu \mathrm{m}$ ) at increasing distances is performed by mounting the device on a Coordinate Measuring Machine (CMM) (Fig 9).

After securing the device to the CMM, the optical axis direction was detected with the help of a marker placed on the centre of the IR image, by assuring that during the vertical translation of the camera the optical centre remains aligned with the marker (see Fig 10).

The calibration of the optical axis of the camera has been assessed by framing the planar surface of the CMM table and verifying that the IR image centre (the superimposed white cross) corresponds to the depth map centre.

The acquisition step was performed exploiting the ability of the high precision machine to move arbitrarily along a single axis, incrementing of $5 \mathrm{~cm}$ each step, relatively to the CMM coordinate system. The systematic distance inhomogeneity brings two main systematic error components increasing with

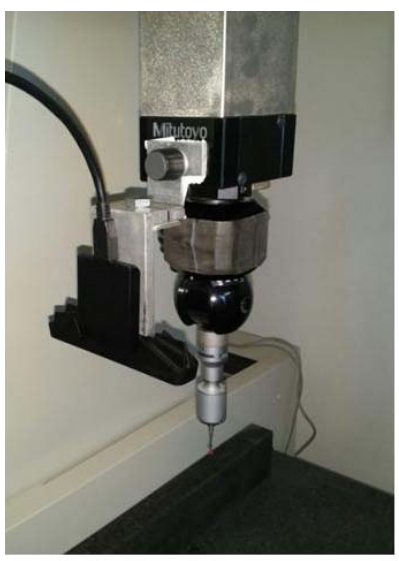

(a)

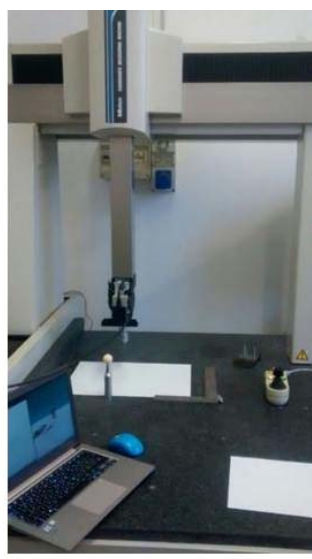

(b)
Fig. 9. The SR300 device mounted on the CMM on the left. The experimental setup on the right.

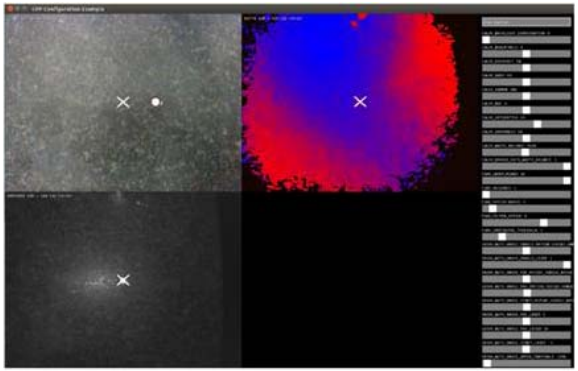

(a)

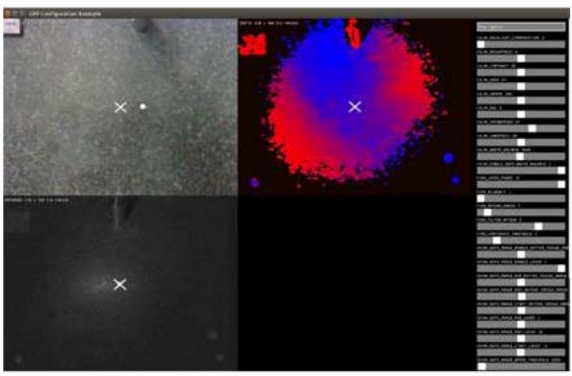

(b)

Fig. 10. The calibration of the optical axis of the camera has been assessed by framing the planar surface of the CMM table and verifying that the IR image centre (the superimposed white cross) corresponds to the depth map centre: (a) close distance $(20 \mathrm{~cm})$ and (b) far distance $(40 \mathrm{~cm})$.

the distance: the non-planarity and the depth offset, as summarized in Figure 11.

We would like to remark that differently from [21] or [27] in which the error is evaluated to be compensated, in this work the aim is to merely characterize it in the selected working field.

1) Systematic Non-Planarity Error Assessment: To decouple the random error from the systematic one, two approaches are described in literature. One foresees to get more acquisitions for each chosen camera position and averaging them [19]. The other, proposed by Guidi et al. [21], consists in filtering out the random error (that it is supposed to occupy the low frequencies) through a smoothing filter.

Inspired by the procedure proposed in [27], as already mentioned in this work the systematic error is evaluated by 


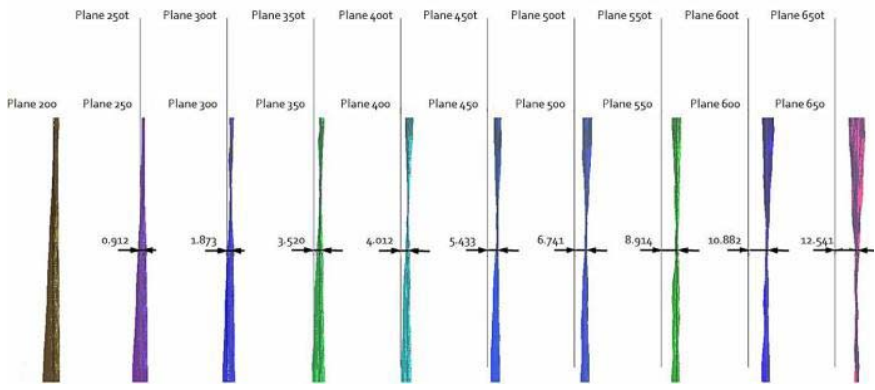

Fig. 11. The systematic distance inhomogeneity brings two main systematic error components increasing with the distance: the non-planarity and the depth offset.
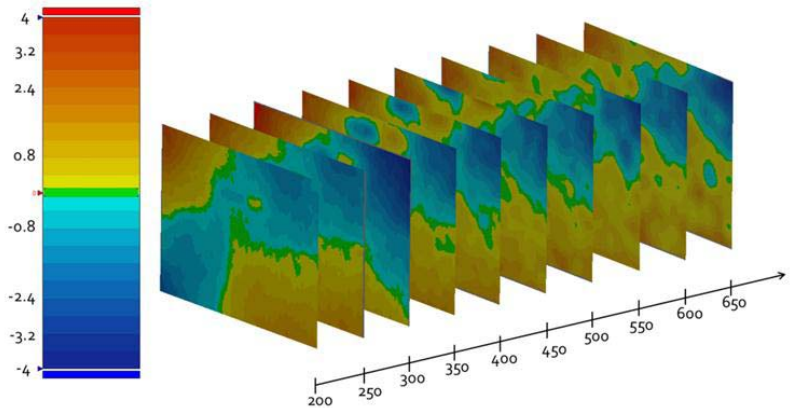

Fig. 12. Error map between the target white plane and the 10 point clouds acquired using the SR300 device with increasing distance from the plane itself.

relying on a precise ground truth to assess the planarity of the reference i.e. by precisely vertically moving the device with respect to the plane. In Figure 12 the flatness error for the 10 point clouds acquired by moving vertically the sensor is depicted.

2) Systematic Depth Offset Assessment: As reported in [23] the configuration of structured light system is "flexible" this meaning that either a single camera (as in the case of PrimeSense products and the Intel ${ }^{\circledR}$ F200 and SR300) or two cameras (as in the case of the Intel ${ }^{\circledR} \mathrm{R} 200$ ) can be used to acquire the scene. However, all devices can be considered as different members of the same family in the unified framework proposed in [28] by which a system with a single camera and an illuminator is equivalent to a system with two rectified cameras and an illuminator.

As such, to determine the theoretical depth estimation error of the SR300 camera we can consider a scenario with two cameras with focal length $f$ separated by distance $b$. Let $d$ be the difference in $x$ coordinates, called disparity, of two corresponding pixels. The depth $z$ of the triangulated point is given by $=\frac{b f}{d}$. The depth error can be rewritten in terms of the disparity error $\varepsilon_{d}$ (when $\varepsilon_{d}$ is small as the pixel size, the approximation holds):

$$
\varepsilon_{z}=\frac{b f}{d}-\frac{b f}{d+\varepsilon_{d}}=\frac{z^{2} \varepsilon_{d}}{b f+z \varepsilon_{d}} \approx \frac{z^{2}}{b f} \cdot \varepsilon_{d}
$$

The final step is obtained by taking the first order Taylor series approximation about $\varepsilon_{d} \approx 0$.

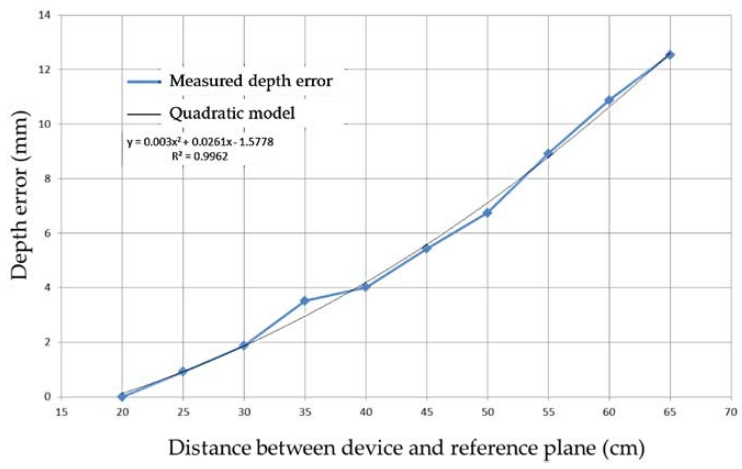

Fig. 13. The depth sensor precision by varying the distance. A quadratic relationship between the distance to the depth camera and the standard deviation of depth values is found.

The experimental data shown in Fig. 13 confirms the expected error model in which $\varepsilon_{z}$ has a quadratic trend in the $z$ direction. The graph, showing how far off the camera measurements are from the average distance, demonstrate that the depth error values for increasing distances can be approximated by a parabolic function with a high coefficient of determination $\left(R^{2}=0.9962\right)$.

\section{Error Assessment Removing the Boundary Edges of the Acquired Data}

Optical depth sensors acquisitions are often prone to a borders degradation, depending on the underlying camera technology; in fact, when the optical ray is tangent with respect to the object to be measured, a little variation of a pixel on the sensor leads to a considerable difference in the detected pixel coordinates. For this reason, in proximity of an edge these devices show strong deformations and cannot be considered fully reliable. As such, and for the sake of completeness, the performances of the camera after excluding the object contours are here further investigated.

For this test, a polystyrene sphere having a diameter of $18 \mathrm{~mm}$, acquired at about $50 \mathrm{~cm}$ from the camera, is considered. Figure 14 shows the obtained results by considering all data (left), and by removing $8 \%$ of the borders from the acquisition (right).

Discarding the borders the span of radial distance decrease remarkably: from $19 \mathrm{~mm}$ to $5.5 \mathrm{~mm}$. However, such results can be noticed on the $P_{F i}$ and $F_{i}$ errors by considering not only the error span of each acquisition, but also the averaged mean and the corresponding standard deviation.

The systematic non-planarity error represented in Figure 15 shows a constant behaviour of the scanner in the central part of the field of view with a maximum deviation from the best fit plane of $\pm 4 \mathrm{~mm}$.

Such an error obviously increases when considering the whole scanned area (at a 650-mm distance), as shown in Figure 15, presenting a maximum deviation of $\pm 11 \mathrm{~mm}$.

\section{E. Multiple Views Performances}

Even if a sub-millimetric precision can be offered by high end laser scanner or photogrammetry techniques for 

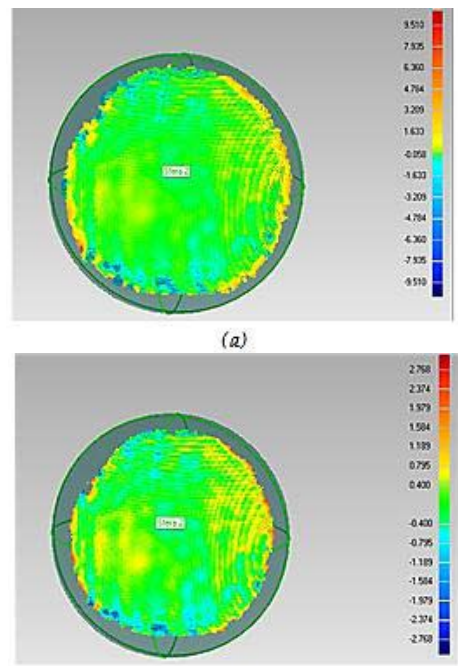

(b)

Fig. 14. Example of radial distance computed between the captured sphere and best-fitted sphere: (a) without deleting noisy boundaries and (b) after boundary elimination.
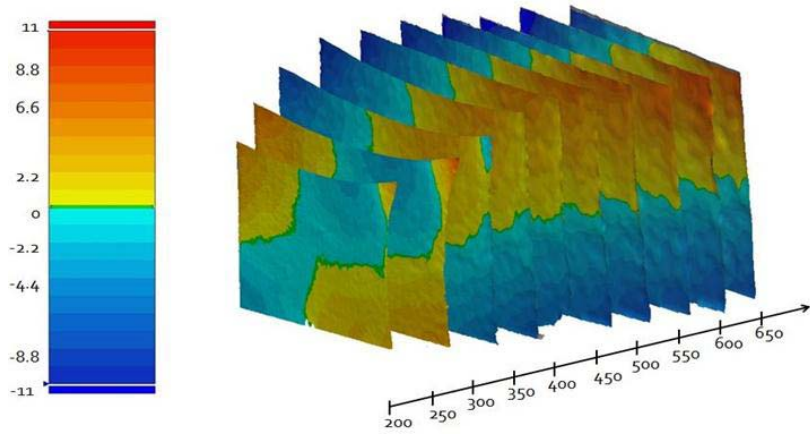

Fig. 15. Error map between the target white plane and the 10 point clouds acquired using the SR300 device with increasing distance from the plane itself, using the whole framed area.

3D reconstruction of small objects, depth cameras used as a multiple views 3D cameras, offer an excellent trade-off between cost and scanning result. To assess the performances of a realistic 3D mesh acquisition, multiple 3D views of the same object to be measured have been generated by positioning the sensor around it.

Finding inspiration by the VDI/VDE Part 3 guidelines on Multiview acquisition - which recommends 5 devices placed at a distance from one another such as to contain the working volume introduced above - in the multiple views experiment, we acquired 5 images for each object, rotating it with respect to a stable camera, placed $0.40 \mathrm{~m}$ far from it.

The SR300 camera multi-views performances have been surveyed over two meaningful objects: 1) a freeform smooth object ( $0.20 \mathrm{~m}$ height and $\sim 0.07 \mathrm{~m}$ wide) and 2$)$ a 3D Tangram object (bounding box with dimensions $0.15 \mathrm{~m}$ height and $0.15 \mathrm{~m}$ wide). To obtain two reference models for assessing the device performance, the Romer Absolute Arm 7520 SI/SE has been used (see Figure 16).

The meshes, obtained using Geomagic $®$ Studio software package (3D Systems, Rock Hill, South Carolina, USA) starting from the point cloud acquired with the SR300 camera, and

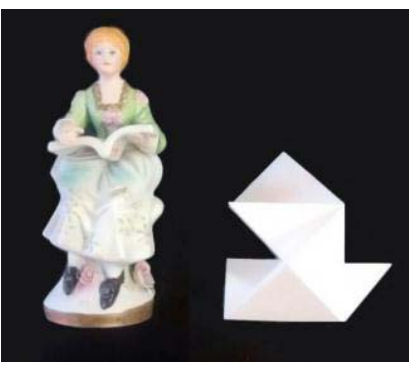

(a)

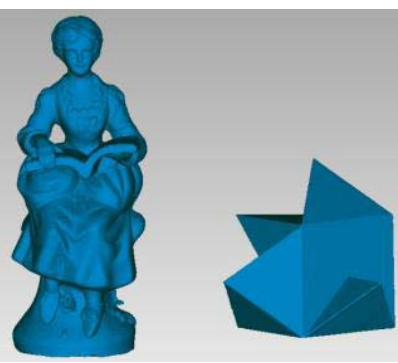

(b)
Fig. 16. (a) Test objects for multiple-view reconstruction: a freeform statue and a 3D tangram; (b) reference 3D models for multiple-view reconstruction, obtained starting from the Romer Absolute Arm acquisition (accuracy of +$0.063 \mathrm{~mm}$ ).
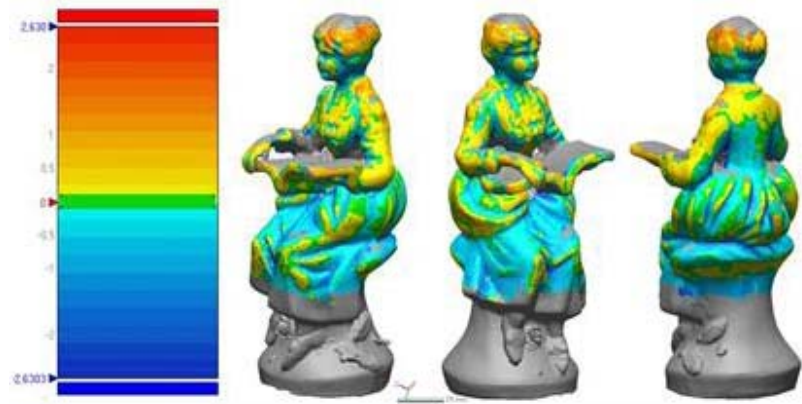

Fig. 17. 3D comparison between the statue ground truth and the SR300 acquired data (mesh). Errors are in $\mathrm{mm}$.
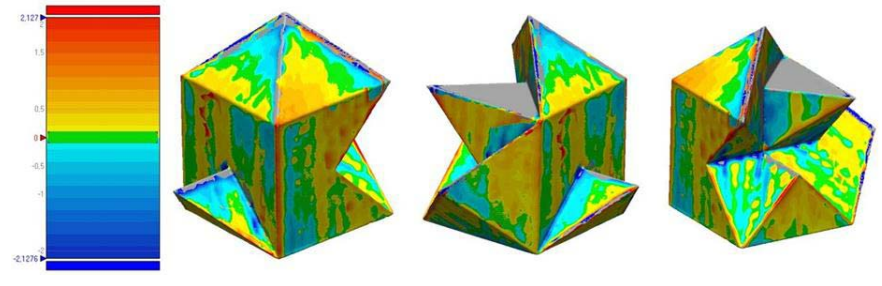

Fig. 18. 3D comparison between the tangram ground truth and the SR300 acquired data (mesh). Errors are in $\mathrm{mm}$.

the reference 3D model have been globally registered using the ICP algorithm.

The Euclidean distances between them are shown in Figures 17 and 18; to increase the quality of the mesh reconstruction, $5 \%$ borders have been removed prior to perform the alignment. The comparison between the ground truth and the acquired data is limited to the target portion obtained with the multiple-view acquisition; grey areas in Figures 17 and 18 are not, therefore, considered.

\section{Discussion AND CONCLUSIONS}

According to experimental results, the SR300 close range camera, when used as a 3D scanner, presents a significant alteration of the captured real world 3D coordinates when the whole scanning volume is considered. However, its performances should be compared with the ones of other RGB-D devices to understand if SR300 is in line with competitor cameras. To this purpose, the results from the analyzed 
TABLE IV

COMPARISON BETwEen SR300, PRIMESENSE CARMINE 1.09 AND KINECT V2 IN TERMS OF VDI/VDE ERROR ASSESSMENT

\begin{tabular}{ccccc}
\hline \hline Device & $\begin{array}{c}\text { SR 300 } \\
\text { filtered data } \\
\text { (reference) }\end{array}$ & Kinect v2 & $\begin{array}{c}\text { Carmine } \\
\mathbf{1 . 0 9}\end{array}$ & $\begin{array}{c}\text { SR300 } \\
\text { (raw data) }\end{array}$ \\
$P_{F}[\mathbf{m m}]$ & 8,3 & 20,13 & 9,32 & 15,43 \\
$\Delta_{P F}[\mathbf{m m}]$ & - & 11,83 & 1,02 & 7,13 \\
$P_{S}[\mathbf{m m}]$ & 1,91 & 3,87 & 9,41 & 4,57 \\
$\Delta_{P S}[\mathbf{m m}]$ & - & 1,96 & 7,5 & 2,66 \\
SS [mm] & 6,05 & 19,7 & 26,08 & 5,11 \\
$\Delta_{S S}$ & - & 13,65 & 20,03 & $-0,94$ \\
$\mathbf{F}[\mathbf{m m}]$ & 6,88 & 12,58 & 6,71 & 19,33 \\
$\Delta_{F}$ & - & 5,7 & $-0,17$ & 12,45 \\
\hline \hline
\end{tabular}

device are compared with those from Primesense Carmine 1.09 and with the Kinect $v 2$. In this work, such a comparison is carried out with reference to the VDI/VDE standards.

Moreover, since a number of 3D scanners have been characterized in literature by using the mentioned normative, it is possible to partially compare the SR300 also with other devices such as, for instance, Smartscan, DPI7 and Structure Sensor. Finally, the multi-view acquisition performed using the tested device is compared with analogous acquisitions carried out by other researchers by using the Kinect $v 2$.

\section{A. VDI/VDE-Based Comparison}

To provide a direct comparison SR300, Primesense Carmine 1.09 and the Kinect $v 2$, the same experimental test setup carried out as described in Sections III.A and III.B is repeated for such competitors. Depth data, acquired with respectively the OpenNI2 library [29] and the Libfreenect2 library [30], are used for evaluating VDI/VDA-based parameters. Results of this test are presented in Table IV where the SR300 with filtered data (i.e. 3D scanning setting for the camera) are used as the reference for comparing the performance. For each of the parameters assessed using the VDI/VDE standard, a parameter $\Delta$ is evaluated as the difference between any normative-based error measured for the SR300 and the same error measured for competitor cameras.

Referring to probing error $P_{F}$, both Primesense Carmine 1.09 and SR300 present comparable results while Kinect $v 2$ is characterized by an higher error. On the other hand, considering the probing error $P_{S}$ is Kinect $v 2$ that performs analogously than the SR300 that, in any case, proves to be the most effective in this test. Higher performance of SR300 is even more evident when dealing with sphere-spacing error SS; in fact, the $\Delta_{S S}$ is considerably higher for tested competitor cameras. The performance in terms of flatness error $\mathrm{F}$ is almost the same for the SR300 and Primesense Carmine 1.09. Interestingly, the behaviour of Kinect $v 2$ in all tests is quite satisfying considering that this camera system is specifically designed to work in medium to long range. Interestingly, the sphere spacing error measured for SR300 using row data is almost the same assessed using optimized settings. This may be due to the fact that the moderate smoothing effect obtained using the optimized setting does not have particular effect on

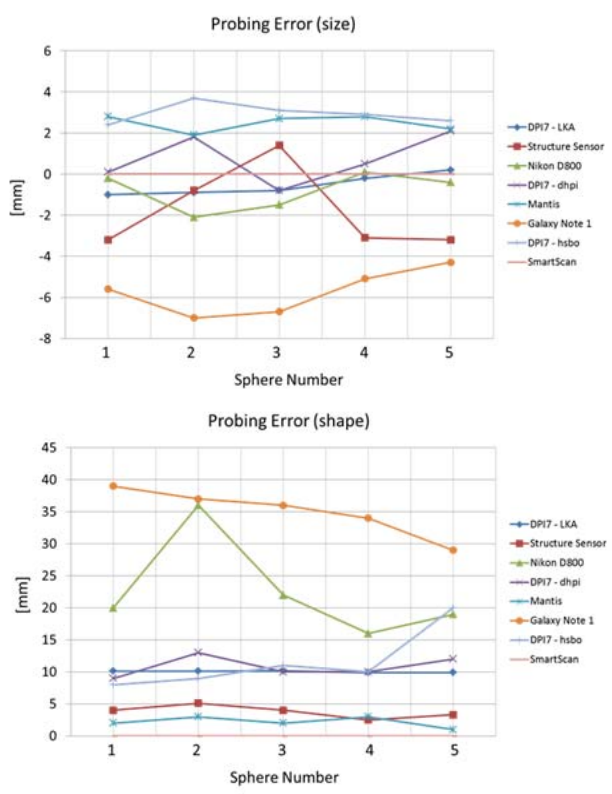

Fig. 19. Results of probing error (PS / PF) equivalent to VDI/VDE 2634, part 2 as reported in [32, Fig. 3].

the position of spheres centrum when compared with the same position evaluated using raw data.

To partially extend the comparison to other similar devices, some results from scientific literature can be used. Referring to probing errors, for instance, the SR300 performance can be compared also to the ones provided in [31] and [32]. In both such contributions, authors evaluate the performance of the sensors by testing more cameras of the same model. In Figure 19 the probing errors (PS / PF) of several depth cameras, evaluated in [32] are shown.

It has to be noticed that among the variety of devices inspected in [32] the most relevant competitor for the SR300 camera the Occipital ${ }^{\circledR}$ Structure Sensor device which basically exploit analogous technology for 3D acquisition.

As demonstrated in Figure 19 the form error presents an average value of $8.3 \mathrm{~mm}$ for the Intel ${ }^{\circledR}$ SR300 filtered data and an average value of 13.93 for raw data, comparable to the $\sim 10 \mathrm{~mm}$ average value measured using the Occipital® sensor. The size error is $1.9 \mathrm{~mm}$ for the filtered data and $4.57 \mathrm{~mm}$ for the raw data, compared with $\sim 2.2 \mathrm{~mm}$.

Referring to sphere spacing error, it is possible to see, in Fig. 20, the deviations observed in [31] and referred to the sphere spacing measurement using the Asus Xtion Pro camera are reported. Such errors, spanning from -8 to $2 \mathrm{~mm}$, are in line with the SR300 performances providing (see Table IV) an average SS error in the range 5-6 $\mathrm{mm}$. Also flatness error can be compared with the one provided in literature [32] and referred to a number of devices (see Fig. 21).

The average performance of SR300 camera (with optimized setting) in terms of flatness, listed in Tab. IV, is lower but "comparable" to the performance of DPI7 3D scanners and is considerably better than the one reported for the Structure Sensor device. Quite the reverse, referring to raw data, SR300 performance is comparable only with the one assessed in literature for the Structure Sensor. 


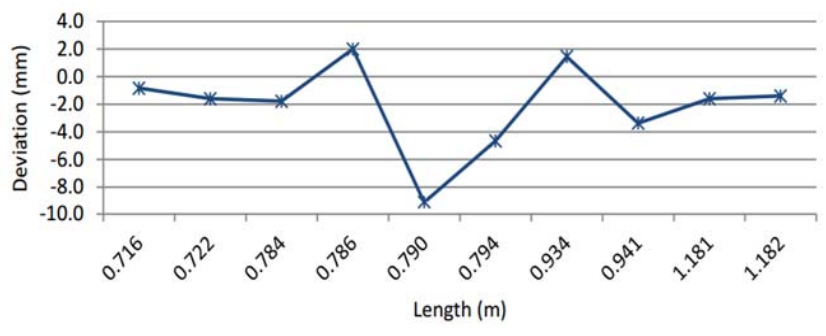

Fig. 20. Results of the deviations in the sphere spacing measurements of the Asus Xtion Pro camera as reported in [31, Fig. 8].

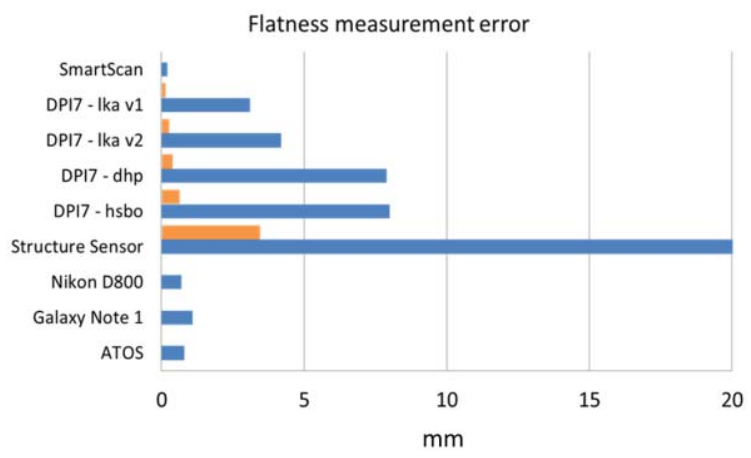

Fig. 21. Results of flatness measurement BLUE - flatness measurement error, ORANGE: standard deviation as reported in [30, Fig. 5].

\section{B. Multi-Views-Based Comparison}

The multi-views acquisition test for the full 3D reconstruction proves (see Figs. 17-18) that the prismatic shape (tangram 3D) is slightly better measured than the free form shape (statue). The error range computed with the latter varies more than the range of the tangram object.

For both the acquired objects the reconstruction results appear to achieve a better precision with respect to the Kinect 2 device tested in [19] for which the error range span in an interval of $27 \mathrm{~mm}$ (as shown in [19, Fig 12b]) compared to the worst case here presented (the free form statue) showing a maximum error lower than $2.5 \mathrm{~mm}$.

\section{Concluding Remarks}

Concluding, test addressed to the Intel ${ }^{\circledR}$ SR300 shows that this device allows to acquire 3D scenes (i.e. to work as a 3D scanner) with a performance comparable to most competitor cameras, or even better when used as a close range 3D scanner, despite its miniaturized dimensions especially when the camera settings are properly optimized.

To further demonstrate that the selected option is effective for using the sensor as a 3D scanner, we measured sphere spacing and probing errors also using all the available filters. Results listed in Table $\mathrm{V}$ prove that in the close range the default filter is the one that outperforms [33].

To reassume the results of our study, we can state that despite the device is born for addressing applications such as tracking, gaming or gesture recognition, it could also be satisfactorily employed as a 3D scanner i.e. could be referred as a low-cost device for a number of 3D scanning applications
TABLE V

Results of The Probing ERror Test (Size AND Form), SPHERE-SPACING USING DIFFERENT FILTERS

\begin{tabular}{ccccc}
\hline \hline $\begin{array}{c}\text { Filter } \\
\text { option } \\
{[\mathbf{3 3}]}\end{array}$ & Description & $\begin{array}{c}\boldsymbol{P}_{\boldsymbol{F}} \\
{[\mathbf{m m}]}\end{array}$ & $\begin{array}{c}\boldsymbol{P}_{\boldsymbol{S}} \\
{[\mathbf{m m}]}\end{array}$ & $\begin{array}{c}\boldsymbol{S S} \\
{[\mathbf{m m}]}\end{array}$ \\
Filter 3 & $\begin{array}{c}\text { Very low smoothing effect with } \\
\text { high sharpness, accuracy levels, and } \\
\text { low noise artefacts. }\end{array}$ & 13.93 & 1.58 & 9.99 \\
Filter 4 & $\begin{array}{c}\text { Low smoothing effect with high } \\
\text { sharpness and accuracy levels. } \\
\text { Moderate smoothing effect, good }\end{array}$ & 12.75 & 2.03 & 10.21 \\
Filter 5 & $\begin{array}{c}\text { Mharpness level, high accuracy and } \\
\text { moderate noise artifacts. }\end{array}$ & 8.30 & 1.91 & 6.05 \\
\hline \hline
\end{tabular}

dealing, for instance, with health, fashion, fitness and Cultural Heritage.

\section{REFERENCES}

[1] (2013). T. Simonite, Depth-Sensing Cameras Head to Mobile Devices, MIT Technology Review's. [Online]. Available: https//:www. technologyreview.com/news

[2] J. Shotton et al., "Real-time human pose recognition in parts from a single depth image," in Proc. IEEE Comput. Soc. Conf. Comput. Vis. Pattern Recognit., Sep. 2011, pp. 1297-1304.

[3] S. Izadi et al., "KinectFusion: Real-time 3D reconstruction and interaction using a moving depth camera," in Proc. ACM Symp. User Interface Softw. Technol., 2011, pp. 559-568.

[4] R. Furferi, L. Governi, F. Uccheddu, and Y. Volpe, "A RGB-D based instant body-scanning solution for compact box installation," in Advances on Mechanics, Design Engineering and Manufacturing. Springer, 2017, pp. 819-828.

[5] Microsoft Kinect, accessed 2016. [Online]. Available: https://msdn. microsoft.com/en-us/library/jj131033.aspx

[6] Primesense, accessed 2016. [Online]. Available: http://www.i3du.gr/pdf/ primesense

[7] Asus. Xtion Pro, accessed 2016. [Online]. Available: http://www. asus.com/Multimedia/Motion_Sensor/Xtion_PRO/

[8] Occipital Structure Sensor, accessed on 2017. [Online]. Available: http://structure.io

[9] ORBBEC. accessed Mar. 2017. [Online]. Available: https:// orbbec3d.com/

[10] Intel RealSense, accessed Mar. 2017. [Online]. Available: www.intel. com/realsense

[11] J.-A. Beraldin, D. Mackinnon, and L. Cournoyer, "Metrological characterization of 3D imaging systems: Progress report on standards developments," in Proc. 17th Int. Congr. Metrol., 2015, p. 13003. [Online]. Available: https://doi.org/10.1051/metrology/20150013003

[12] VDI/VDE 2634 Blatt 2, Optische 3D-Messsysteme-Systeme mit flachenhafter Antastung/Optical 3-D Measuring Systems-Optical Systems Based on Area Scanning, 11 pages, Aug. 2002. Blatt 3, Optische 3D-Messsysteme-Bildgebende Systeme mit flächenhafter Antastung in mehreren Einzelansichten/Optical 3D-Measuring Systems-Multiple View Systems Based on Area Scanning, Sep. 2006.

[13] Z. Zhang, "Iterative point matching for registration of free-form curves and surfaces," Int. J. Comput. Vis., vol. 13, no. 2, pp. 119-152, 1994.

[14] H. Gonzalez-Jorge et al., "Metrological comparison between Kinect I and Kinect II sensors," Measurement," vol. 70, pp. 21-26, Jun. 2015, doi: 10.1016/j.measurement.2015.03.042.

[15] O. Wasenmüller and D. Stricker, "Comparison of Kinect v1 and v2 depth images in terms of accuracy and precision," in Proc. Asian Conf. Comput. Vis. Workshop (ACCV Workshop), 2016, pp. 1-12.

[16] S. Zennaro et al., "Performance evaluation of the 1st and 2nd generation Kinect for multimedia applications," in Proc. IEEE Int. Conf. Multimedia Expo (ICME), Jul. 2015, pp. 1-6, doi: 10.1109/ICME.2015.7177380.

[17] K. Khoshelham and S. O. Elberink, "Accuracy and resolution of Kinect depth data for indoor mapping applications," Sensors, vol. 12, no. 2, pp. 1437-1454, 2012, doi:10.3390/s120201437.

[18] T. Dutta, "Evaluation of the Kinect sensor for 3-D kinematic measurement in the workplace," Appl. Ergonom., vol. 43, no. 4, pp. 645-649, Jul. 2012. [Online]. Available: http://dx.doi.org/10.1016/ j.apergo.2011.09.011

[19] E. Lachat, H. Macher, T. Landes, and P. Grussenmeyer, "Assessment and calibration of a RGB-D camera (Kinect v2 sensor) towards a potential use for close-range 3D modeling," Remote Sens., vol. 7, no. 10, pp. 13070-13097, 2015, doi:10.3390/rs71013070. 
[20] T. Breuer, C. Bodensteiner, and M. Arens, "Low-cost commodity depth sensor comparison and accuracy analysis," Proc. SPIE, vol. 9250, p. $92500 \mathrm{G}$, Oct. 2014 , doi: 10.1117/12.2067155.

[21] G. Guidi, S. Gonizzi, and L. Micoli, "3D capturing performances of low-cost range sensors for mass-market applications," in Proc. ISPRSInt. Arch. Photogramm., Remote Sens. Spatial Inf. Sci., 2016, pp. 33-40.

[22] L. Nhuy. A Comparison of Intel RealSense Front-Facing Camera SR300 and F200, accessed 2017. [Online]. Available: https://software. intel.com/en-us/articles/a-comparison-of-intel-realsensetm-front-facingcamera-sr300-and-f200

[23] P. Zanuttigh, G. Marin, C. Dal Mutto, F. Dominio, L. Minto, and G. M. Cortelazzo, "Operating principles of structured light depth cameras," Time-of-Flight and Structured Light Depth Cameras: Technology and Applications. Springer, 2016, pp. 43-79. [Online]. Available: http://dx.doi.org/10.1007/978-3-319-30973-6_2

[24] F. Menna, F. Remondino, R. Battisti, and E. Nocerino, "Geometric investigation of a gaming active device," Proc. SPIE, vol. 8085, p. 80850G, Jun. 2011, doi: 10.1117/12.890070.

[25] Micro-mirrors from ST Microelectronics Provide Precision in Perceptual Computing Adoption of Micro-Mirrors Helps Fundamentally Change Human-Computer Interaction, Geneva, accessed on Mar. 2, 2015. [Online]. Available: http://www.st.com/content/st_com/en/about/mediacenter/press-item.html/p3657.html

[26] Librealsense, accessed Mar. 2017. [Online]. Available: https://github. com/IntelRealSense/librealsense

[27] F. Chiabrando, R. Chiabrando, D. Piatti, and F. Rinaudo, "Sensors for 3D imaging: Metric evaluation and calibration of a CCD/CMOS timeof-flight camera," Sensors, vol. 9, no. 12, pp. 10080-10096, 2009.

[28] J. Davis, D. Nehab, R. Ramamoorthi, and S. Rusinkiewicz, "Spacetime stereo: A unifying framework for depth from triangulation," in Proc. IEEE Conf. Comput. Vision Pattern Recognit., Sep. 2003, pp. II-359-II-366.

[29] OpenNi2, accessed Mar. 2017. [Online]. Available: https://github.com/ OpenNI/OpenNI2

[30] Libfreenect2, accessed Mar. 2017. [Online]. Available: https://github. com/OpenKinect/libfreenect2

[31] J. Boehm, "Accuracy investigation for structured-light based consumer 3D sensors," in Proc. Photogramm.-Fernerkundung-Geoinf., 2014, pp. 117-127.

[32] T. P. Kersten, H.-J. Przybilla, M. Lindstaedt, F. Tschirschwitz, and M. Misgaiski-Hass, "Comparative Geometrical Investigations of HandHeld Scanning Systems," in Proc. ISPRS-Int. Arch. Photogramm., Remote Sens. Spatial Inf. Sci., 2016, pp. 507-514.

[33] Accessed on Mar. 2017. [Online]. Available: https://software.intel.com/ sites/landingpage/realsense/camera-sdk/v1.1/documentation/html/index. html?setivcamfilteroption_device_pxccapture.html

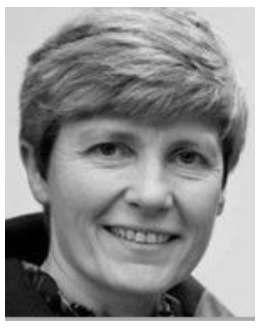

Monica Carfagni received the Ph.D. degree. She is currently a Full Professor with the Department of Industrial Engineering of Florence, University of Florence, Italy, where she teaches the courses Mechanical Drafting, Computational Graphics, and Additive Manufacturing with the School of Engineering. She has authored more than 100 papers in conferences and journals. Her main research activities are on computer-aided design, image processing, industrial diagnostics, virtual prototyping, FE simulation, and computer-aided tolerancing.

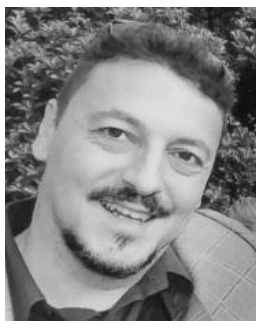

Rocco Furferi received the Ph.D. degree. He is currently an Assistant Professor with the Department of Industrial Engineering, University of Florence, Italy, where he teaches the course Mechanical Drafting. His main research activities are computer-based methods and tools, 2-D and 3-D machine vision for industrial process and product control and inspection, geometric modeling, $\mathrm{CAD}$, and computational graphics. In these areas, he has authored more than 70 publications in scientific journals and refereed conference proceedings.

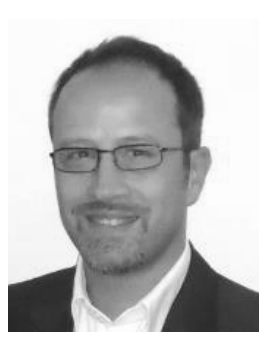

Lapo Governi received the Ph.D. degree. He is currently an Associate Professor with the Department of Industrial Engineering, University of Florence, Italy, where he teaches the courses Reverse Engineering and Rapid Prototyping with the School of Engineering. His research work is mainly focused on 3-D modeling and computational geometry, image processing and computer vision, reverse engineering, virtual prototyping, shape and structural optimization, industrial design, and computer-aided tolerancing. In these areas, he has authored over 90 publications in scientific journals and refereed conference proceedings.

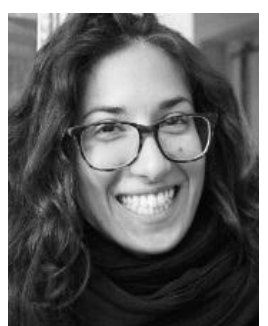

Michaela Servi received the B.S. degree from the University of Florence, Italy in 2012, and the M.S. degree from the University of Florence, in 2015. She is currently pursuing the Ph.D. degree with the Department of Industrial Engineering from the same University.

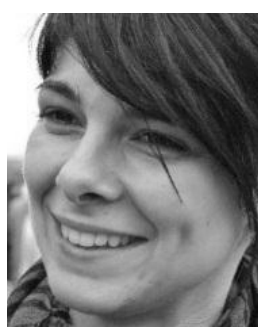

Francesca Uccheddu received the Ph.D. degree. She is currently a Researcher with the University of Florence. In her work, she pioneered the use of the 3-D wavelets analysis for inventing a new watermarking algorithm for 3-D models. She is with the Department of Industrial Engineering and is currently focused on the development of 3-D processing algorithms for biomedical applications. Her research activities span from 3-D processing to reverse engineering to image processing and computer vision.

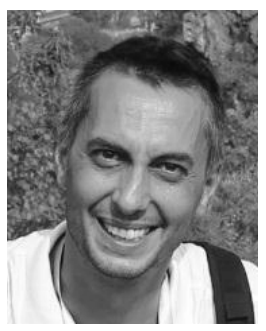

Yary Volpe received the $\mathrm{Ph} . \mathrm{D}$. degree. He is currently an Assistant Professor with the Department of Industrial Engineering, University of Florence, Italy, where he teaches the course CAD Modeling with the School of Engineering. He has authored more than 50 journal papers and refereed conference proceedings. His research work is addressed to 3-D modeling, mechanical design, computational geometry, human-computer interaction, and reverse engineering. 